\title{
Developing An Interactive Game Platform to Promote Learning and Teamwork on Mobile Devices: An Experience Report
}

\author{
Vincent Tam ${ }^{*}$, Z.X. Liao , Alvin C.M. Kwan ${ }^{* *}$, C.H. Leung ${ }^{*}$ and Lawrence K. Yeung ${ }^{*}$ \\ *Department of Electrical \& Electronic Engineering, The University of Hong Kong, Hong Kong. \\ ${ }^{* *}$ Faculty of Education, The University of Hong Kong, Hong Kong. \\ E-mail:vtam@eee.hku.hk
}

\begin{abstract}
In the past few years, many new development toolkits such as the Nebula2 and/or mobile technologies including the WiFi or mobileTV have opened up exciting learning opportunities on mobile devices. On top of it, new technologies continue to fuel the rapid growth of newly merged fields of research like the edutainment for educational entertainment. In a recent teaching development project, we have developed an interactive game platform to facilitate learning and more importantly the spirit of teamwork for collaborative problem-solving on desktop and pocket PCs. With the great challenges imposed by globalization, we strongly believe that learning to collaboratively analyze and then apply the "appropriate" knowledge to solve a specific problem is always the key to success. In this paper, we discuss about an on-going work, and share our relevant experience in system development. Furthermore, evaluation strategies will be thoroughly examined. After all, our work shed light on many interesting directions for future exploration.
\end{abstract}

\section{Introduction}

Undoubtedly, new telecommunication technologies or services including the High-Speed Downlink Packet Access (HSDPA), namely the 3.5G, the Wireless Fidelity (WiFi) technologies for the interoperability of the IEEE 802.11 based products, or lately the mobileTV, have been continuously reshaping our modern living. With the availability of powerful mobile devices connected to a high-speed wireless network, with the maximum data rate as several to tens of megabits per seconds nowadays, many attractive mobile learning applications realizing the concept of learning anytime and anywhere have been developed in recent years, and actively sought the world-wide attention of educators, students, lifelong learners or professionals in various disciplines. Among many successful applications, the Cellphedia [1] is a Mobile Social Software (MoSoSo) that promotes the sharing of knowledge, in which users can send and receive encyclopedia-type inquiries between specific, predefined groups of users through text messaging. Besides, the United Kingdom initiated the eVIVA [3] project that aimed to assess the key stage 3 students' performance in Information and Communications Technology (ICT) [9] using the latest mobile technologies. In addition to the uses of mobile devices for learning or assessment, new technologies continue to fuel the blending and rapid development of new fields of research such as the edutainment for educational entertainment. An example is the digital game based learning (DGBL) approach [4] advocated by M. Prensky, that combines serious learning and interactive entertainment [8] in a fun, engaging and highly exciting medium.

In a Faculty-supported Teaching Development Project, we proposed to develop an interactive game platform based on a virtual university campus containing game rooms with various missions for different groups of students to complete on wireless mobile devices so as to enhance the learners' experience after classes. All the missions, in different forms of mini-games, are focused on engaging players to exercise their logical thinking and collaborative problem-solving skills relevant to specific Engineering disciplines. To promote the spirit of teamwork as in [8], each team of 2 to 3 students will work together to complete all the missions in order to compete for top ranks among other teams in the virtual campus. As our interactive game can be accessed through wireless mobile devices such as pocket PCs, each team can easily continue its missions in the game anytime and 
anywhere. To demonstrate the feasibility of our proposal, we used the Nebula Version 2.0 toolkit to build a prototype of our interactive game containing various game rooms that can be accessed through Window-based pocket PCs. When the prototype is completed, a detailed evaluation will be conducted to analyze the effectiveness of our simulation game on motivating and/or enhancing the learners' experience in relevant Engineering disciplines. After all, our work prompts for many interesting directions for further investigations.

This paper is organized as follows. Section 2 details the system architecture design of our interactive simulation game to enhance learners' experience on mobile devices. We give an empirical evaluation of our proposed system on various criteria in Section 3. Lastly, we summarize our work and shed lights on future directions in Section 4.

\section{System Architecture}

Essentially, the whole software system of our interactive simulation game is composed of two major parts: the game engine which deals with high-level game logic, and the Nebula2 kernel which handles lowlevel functions such as graphics, keyboard/mouse inputs, etc. The game engine has three components: game entity, scenario loader and message pump. Each game entity represents an object in the game. By attaching different property to a game entity, the game entity can behave differently. For example, with a physics property, the game entity can collide with other entity whereas without it, the game entity could simply go through. When events arrive, a message dispatcher of game entity is responsible to pass them to appropriate property handler. Game entities in a particular scenario are managed by an entity manager created by scenario loader. The game engine is event driven. For the specific details about the Nebula2 kernel, refer to [5].

\section{Prototype Implementation \& Evaluation}

To demonstrate the feasibility of our proposal, we used the latest Nebula2 toolkit to build a prototype of our interactive game containing several mini-game rooms inside a virtual campus that can be accessed through Windows-based desktop or pocket PCs. The uses of mini-games like robot-shooting or duct/path connection game are aimed to introduce some funs and also flexibility in the design of each game room that can be an individual component by itself, or a spot within an adventure featured by a number of mini-game rooms focusing on a specific subject/topic. In our prototype implementation, we built an adventure of 3 mini-game rooms inside the virtual campus, that try to emphasize on relevant subjects in Information and Communication Technology (ICT) for Engineering students in general. The relevant subjects in ICT we chose for the mini-game rooms include the Basic Organization of Computers, the (more advanced) Structure of Microcomputers and lastly the Application Programming on Computers. Clearly, given the flexibility of our system architecture, relevant subjects in ICT such as the Database Management and User Interface Design, or other different topics can easily be integrated into the virtual campus of our simulation game.

To play the simulation game, each team of 2 to 3 students should "individually" log in the game server via a networked PC and then select to enter into a game room to perform some designated task. Among the team members, the players should nominate one student as the team leader to submit the final decision to each question/task. The team members are allowed to use the text-based voting mechanism or voice using the peer-to-peer Session Initiation Protocol (SIP) to communicate with each other before making any decision so as to encourage teamwork. After completing a set of questions/tasks, each team will be given a score, based on their time and accuracy, which will be added to their overall score. The team with the highest overall score is the winner. Fig. 1 shows the "virtual campus" of our TDG game.

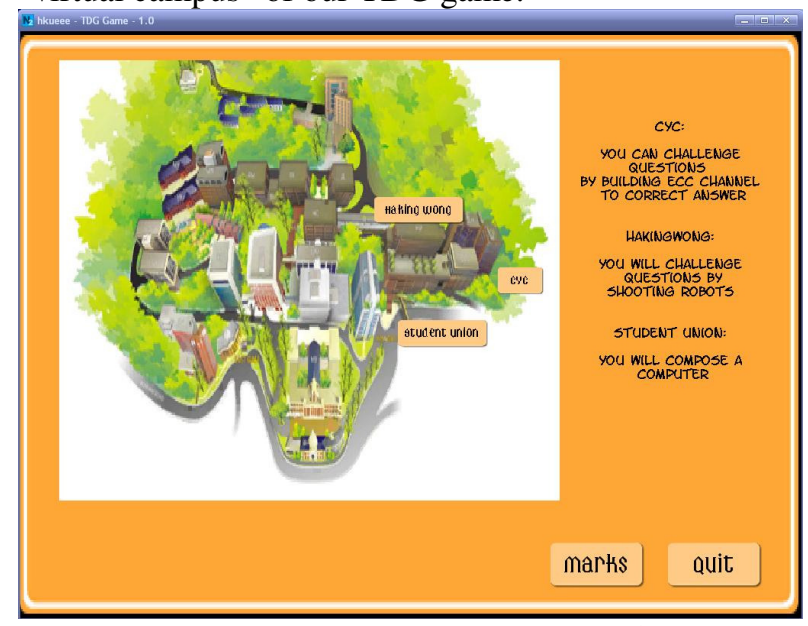

Fig. 1. The virtual campus of our TDG game

As shown in the diagram, the overall scores of all the participating teams can be checked inside the virtual campus from time to time to encourage constructive competition among the teams. For example, in the mini-game room for the Basic Organization of Computers, each team leader will act as a coordinator to collect opinions from other team members via voice 
communication over the SIP server, and then make the necessary decisions to obtain a computer configuration for online submission. Our Nebula2 game engine will proceed to check if it contains all the essential components such as the processor and RAM for a working computer. Accordingly, it will send a message to congratulate the team for their good work. Otherwise, an error message will be displayed to allow the whole team to revise their configuration for later submissions.

Fig. 3 shows the similar interface of our TDG game ported onto the Dell ${ }^{\mathrm{TM}}$ Axim X41v pocket PC installed with an Intel(R) processor of $624 \mathrm{Mhz}$, and the Windows Mobile 5.0 operating system.

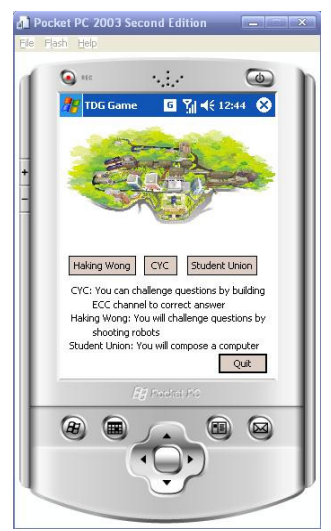

Fig. 3. The pocket PC version of our TDG game

When the above prototypes are completed with extensive testing, a detailed evaluation will be conducted to analyze the effectiveness of our simulation game on motivating and/or enhancing the learners' experience in relevant Engineering disciplines. In particular, students in selected courses such as our level-2 course for Human-Computer Interaction will be divided into two groups of students in which teams in the first group will be given with 'trials' on pocket PCs whereas the remaining is the control group. After that, the students' performance in these two groups will be thoroughly compared and analyzed.

\section{Concluding Remarks}

In this paper, we discuss about an on-going teaching development project that aims to develop an interactive game platform in order to facilitate learning and more importantly the spirit of teamwork for collaborative problem-solving on desktop and pocket PCs. We share our relevant experience in system development. Furthermore, evaluation strategies are thoroughly considered.

After all, there are many interesting directions for further investigations. The integration of our game with existing e-learning systems, the latest Web 2.0 technology or powerful search engines is worth researching.

\section{References}

[1] The Cellphedia development team, The Cellphedia website, maintained by L. Garcia, Retrieved: November 12, 2007, from http://www.cellphedia.com.

[2] The ComputerWorld project team, The Wireless Trends and Technologies Top Stories website, maintained by the IDG computer team, Retrieved: January 15, 2008, from http://www.computerworld.com/taxonomy/000/000/000//taxo nomy_000000078_index.jsp.

[3] The eVIVA project team, The eVIVA project website, maintained by Qualification and Curriculum Authority of the United Kingdom, Retrieved: November 15, 2007, from http://www.qca.org.uk/qca_5962.aspx.

[4] M. Prensky, "Digital Game Based Learning", New York: The twitchspeed.com. Retrieved: June 13, 2007. from http://www.twitchspeed.com/site/news.html.

[5] The Nebula development team, The Nebula Device website, maintained by the Wikipedia team, Retrieved: January 18, 2008, from http://nebuladevice.cubik.org/.

[6] The Sims development team, The Sims official website, Retrieved: January 15, 2008, from http://thesims.ea.com/.

[7] L.S. Vygotsky, "Mind in society", Cambridge, M.A. Harvard University Press, 1978.

[8] J. Ward and P. Carroll, "Can the Use of a Computer Simulation Game Enhance Mechanical Reasoning Ability: an exploratory study", Working Paper Series MCE-0998, 1-20. Retrieved: June 10, 2007, from http://citeseer.ist.psu.edu/431172.html.

[9] The Wikipedia development team, The Information Technology - Wikipedia webpage, maintained by the Wikipedia team, Retrieved: November 18, 2007, from http://en.wikipedia.org/wiki/Information_technology. 